\title{
Loop-Mediated Isothermal Amplification for Screening Salmonella in Animal Food and Confirming Salmonella from Culture Isolation
}

\author{
Kelly J. Domesle ${ }^{1}$, Shenia R. Young ${ }^{1}$, Qianru Yang ${ }^{1}$, Beilei Ge $^{1}$ \\ ${ }^{1}$ Center for Veterinary Medicine, U.S. Food and Drug Administration
}

\section{Corresponding Author}

Beilei Ge

Beilei.Ge@fda.hhs.gov

\section{Citation}

Domesle, K.J., Young, S.R., Yang, Q., $\mathrm{Ge}, \mathrm{B}$. Loop-Mediated Isothermal Amplification for Screening Salmonella in Animal Food and Confirming Salmonella from Culture Isolation. J. Vis. Exp. (159), e61239, doi:10.3791/61239 (2020).

\section{Date Published}

May 20, 2020

DOI

$10.3791 / 61239$

URL

jove.com/video/61239

\section{Abstract}

Loop-mediated isothermal amplification (LAMP) has emerged as a powerful nucleic acid amplification test for the rapid detection of numerous bacterial, fungal, parasitic, and viral agents. Salmonella is a bacterial pathogen of worldwide food safety concern, including food for animals. Presented here is a multi-laboratory-validated Salmonella LAMP protocol that can be used to rapidly screen animal food for the presence of Salmonella contamination and can also be used to confirm presumptive Salmonella isolates recovered from all food categories. The LAMP assay specifically targets the Salmonella invasion gene (invA) and is rapid, sensitive, and highly specific. Template DNAs are prepared from enrichment broths of animal food or pure cultures of presumptive Salmonella isolates. The LAMP reagent mixture is prepared by combining an isothermal master mix, primers, DNA template, and water. The LAMP assay runs at a constant temperature of $65^{\circ} \mathrm{C}$ for $30 \mathrm{~min}$. Positive results are monitored via realtime fluorescence and can be detected as early as $5 \mathrm{~min}$. The LAMP assay exhibits high tolerance to inhibitors in animal food or culture medium, serving as a rapid, reliable, robust, cost-effective, and user-friendly method for screening and confirming Salmonella. The LAMP method has recently been incorporated into the U.S. Food and Drug Administration's Bacteriological Analytical Manual (BAM) Chapter 5.

\section{Introduction}

Loop-mediated isothermal amplification (LAMP) is a novel isothermal nucleic acid amplification test (iNAAT) invented in 2000 by a group of Japanese scientists ${ }^{1}$. Through the formation of a target-specific stem-loop DNA structure during initial steps, LAMP uses a strand-displacing DNA polymerase to efficiently amplify this starting material quasi- exponentially, resulting in $10^{9}$ copies of target in less than 1 $h^{1}$. Compared to polymerase chain reaction (PCR), a widely used NAAT, LAMP possesses several advantages. First, LAMP reactions are carried out under isothermal conditions. This obviates the need for a sophisticated thermal cycling instrument. Second, LAMP is highly tolerant to culture media 
and biological substances ${ }^{2}$ with robustness demonstrated for both clinical and food applications $s^{3,4}$. This simplifies sample preparation and minimizes false negative results ${ }^{5}$. Third, LAMP is amenable to multiple detection platforms, such as turbidity, colorimetry, bioluminescence, fluorescence, and microfluidics ${ }^{6}$. Fourth, LAMP is highly specific as it uses four to six specially designed primers to target six to eight specific regions ${ }^{1,7}$. Fifth, LAMP is ultrasensitive and numerous studies have reported its superior sensitivity to $\mathrm{PCR}$ or real-time $\mathrm{PCR}^{8}$. Finally, LAMP is faster with many assays now adopting a 30 min standard run time while PCRtype assays usually take $1-2 h^{8}$.

These attractive features fueled the application of LAMP in broad pathogen detection areas, including in vitro diagnostics $^{9}$, animal disease diagnostics ${ }^{10}$, and food and environmental testing ${ }^{11}$. Notably, a TB-LAMP (LAMP for Mycobacterium tuberculosis) has been recommended by $\mathrm{WHO}$ as a valid replacement test for sputumsmear microscopy for pulmonary tuberculosis diagnoses in peripheral settings ${ }^{12}$. LAMP application also expands beyond microbial identification to include the detection of allergens, animal species, drug resistance, genetically modified organisms, and pesticides ${ }^{13}$.

Nontyphoidal Salmonella is a zoonotic pathogen of substantial food safety and public health concern worldwide $^{14}$. It has also been identified as an important microbial hazard in food for animals (i.e., animal food ${ }^{15,16}$. To prevent Salmonella illnesses/outbreaks from contaminated human food and animal food, it is imperative to have rapid, reliable, and robust methods for testing Salmonella in a variety of matrices. In the past decade, considerable efforts have been made internationally on the development and application of Salmonella LAMP assays in a wide array of food matrices, as recently summarized in an extensive review ${ }^{8}$. Several Salmonella LAMP assays, including the one presented here, have successfully completed multi-laboratory validation following well-established international guidelines ${ }^{17,18,19,20}$.

Our Salmonella LAMP assay specifically targets the Salmonella invasion gene invA (GenBank accession number M90846) $)^{21}$ and is rapid, reliable, and robust in multiple food matrices $^{4,22,23,24,25,26}$. The method has been validated in six animal food matrices in a precollaborative study ${ }^{26}$ and in dry dog food in a multi-laboratory collaborative study ${ }^{19}$. As a result, the Salmonella LAMP method presented here has recently been incorporated into the U.S. Food and Drug Administration (FDA)'s Bacteriological Analytical Manual (BAM) Chapter 5 Salmonella ${ }^{27}$ to serve two purposes, one as a rapid screening method for the presence of Salmonella in animal food and two as a reliable confirmation method for presumptive Salmonella isolated from all foods.

\section{Protocol}

NOTE: A LAMP reaction mix contains DNA polymerase, buffer, $\mathrm{MgSO}_{4}$, dNTPs, primers, DNA template, and water. The first four reagents are contained in an isothermal master mix (Table of Materials). Primers are premixed in-house to become a primer mix (10x). DNA templates can be prepared from enrichment broths of animal food samples for screening purpose or from cultures of presumptive Salmonella isolates for confirmation purpose. In addition, a positive control (DNA extracted from any Salmonella reference strains, e.g., Salmonella enterica serovar Typhimurium ATCC 19585 [LT2]) and a no template control (NTC; sterile molecular grade water) are included in every LAMP run. 


\section{Preparation of DNA templates}

1. To prepare DNA templates from animal food enrichments, follow these steps.

1. Aseptically weigh $25 \mathrm{~g}$ of animal food sample (e.g., dry cat food, dry dog food, cattle feed, horse feed, poultry feed, and swine feed) into a sterile filter bag (Table of Materials), or equivalent. Place the bag into a large container or rack for support during incubation.

2. Add $225 \mathrm{~mL}$ of sterile buffered peptone water (BPW). Mix well by swirling and brief hand-massage. Let stand at room temperature for $60 \pm 5 \mathrm{~min}$.

3. Mix well by swirling and determine $\mathrm{pH}$ with a test paper. Adjust $\mathrm{pH}$, if necessary, to $6.8 \pm 0.2$ with sterile $1 \mathrm{~N} \mathrm{NaOH}$ or $1 \mathrm{~N} \mathrm{HCl}$. Incubate at $35 \pm 2{ }^{\circ} \mathrm{C}$ for $24 \pm 2 \mathrm{~h}$.

4. Mix well by swirling the bag containing animal food enrichment broths. Transfer $1 \mathrm{~mL}$ from the filtered side of the bag to a microcentrifuge tube. Vortex briefly.

5. Extract DNA using a sample preparation reagent (Table of Materials) as follows.

1. Centrifuge at $900 \times g$ for $1 \mathrm{~min}$ to remove large particles and transfer supernatant to a new microcentrifuge tube.

2. Centrifuge at $16,000 \times g$ for $2 \mathrm{~min}$ and discard supernatant.

3. Suspend the pellet in $100 \mu \mathrm{L}$ of the sample preparation reagent and heat at $100 \pm 1{ }^{\circ} \mathrm{C}$ for 10 min in a dry heat block.
4. Cool to room temperature and store sample DNA extracts at $-20^{\circ} \mathrm{C}$.

2. To prepare DNA templates from presumptive Salmonella cultures, follow these steps.

1. Obtain presumptive Salmonella isolates from culture isolation in all foods following FDA's BAM Chapter 5 Salmonella section D: Isolation of Salmonella ${ }^{27}$.

2. Inoculate presumptive Salmonella isolates on a nonselective agar plate (e.g., blood agar, nutrient agar, and trypticase soy agar) and incubate at $35 \pm$ $2{ }^{\circ} \mathrm{C}$ for $24 \pm 2 \mathrm{~h}$.

3. Transfer several single colonies to $5 \mathrm{~mL}$ of trypticase soy broth (TSB) or brain heart infusion (BHI) broth and incubate at $35 \pm 2{ }^{\circ} \mathrm{C}$ for $16 \pm 2 \mathrm{~h}$.

NOTE: This step can be optional if the presumptive Salmonella culture is pure. In that case, DNA templates can be prepared by suspending several single colonies in $5 \mathrm{~mL}$ of TSB and heat $500 \mu \mathrm{L}$ of the suspension at $100 \pm 1{ }^{\circ} \mathrm{C}$ for $10 \mathrm{~min}$ in a dry heat block. Continue with step 5 below.

4. Transfer $500 \mu \mathrm{L}$ of the overnight culture to a microcentrifuge tube and heat at $100 \pm 1{ }^{\circ} \mathrm{C}$ for 10 min in a dry heat block.

5. Cool to room temperature and store isolate DNA extracts at $-20^{\circ} \mathrm{C}$.

3. To prepare positive control DNA, follow similar steps as above for preparing DNA templates from presumptive Salmonella cultures with one extra dilution step.

1. Inoculate S. Typhimurium ATCC 19585 (LT2) or any Salmonella reference strains on a nonselective agar plate (e.g., blood agar, nutrient agar, and trypticase soy agar) and incubate at $35 \pm 2{ }^{\circ} \mathrm{C}$ for $24 \pm 2 \mathrm{~h}$. 
2. Transfer several single colonies to $5 \mathrm{~mL}$ of TSB or $\mathrm{BHI}$ broth and incubate at $35 \pm 2{ }^{\circ} \mathrm{C}$ for $16 \pm 2 \mathrm{~h}$ to reach $\sim 10^{9} \mathrm{CFU} / \mathrm{mL}$.

3. Serially dilute the overnight culture in $0.1 \%$ peptone water to obtain $\sim 10^{7} \mathrm{CFU} / \mathrm{mL}$.

4. Transfer $500 \mu \mathrm{L}$ of this dilution to a microcentrifuge tube and heat at $100 \pm 1{ }^{\circ} \mathrm{C}$ for $10 \mathrm{~min}$ in a dry heat block.
5. Cool to room temperature and store positive control DNA at $-20^{\circ} \mathrm{C}$.

\section{Preparation of primer mix (10x)}

1. Obtain commercially synthesized LAMP primers (Sal4F3, Sal4-B3, Sal4-FIP, Sal4-BIP, Sal4-LF, and Sal4-LB) with standard desalting purification (Table 1).

\begin{tabular}{|c|c|c|c|}
\hline Primer name & Description & Sequence $\left(5^{\prime}-3^{\prime}\right)$ & Length (bp) \\
\hline Sal4-F3 & Forward outer primer & GAACGTGTCGCGGAAGTC & 18 \\
\hline Sal4-B3 & Backward outer primer & CGGCAATAGCGTCACCTT & 18 \\
\hline Sal4-FIP & Forward inner primer & $\begin{array}{l}\text { фCGCGGCATCCGCATCAATA } \\
\text { TCTGGATGGTATGCCCGG }\end{array}$ & 38 \\
\hline Sal4-BIP & Backward inner primer & $\begin{array}{l}\text { GCGAACGGCGAAGCGTACTG } \\
\text { TCGCACCGTCAAAGGAAC }\end{array}$ & 38 \\
\hline Sal4-LF & Loop forward primer & $\begin{array}{c}\text { TCAAATCGGCATCAATACTCA } \\
\text { TCTG }\end{array}$ & 25 \\
\hline Sal4-LB & Loop backward primer & AAAGGGAAAGCCAGCTTTAC $\$$ & 21 \\
\hline
\end{tabular}

Table 1: LAMP primers for screening Salmonella in animal food and confirming Salmonella from culture isolation. The primers are designed based on the Salmonella invA sequence (GenBank accession number M90846).

2. Prepare stock solutions of each primer $(100 \mu \mathrm{M})$ by rehydrating the primer with appropriate amount of sterile molecular grade water. Mix well by vortexing for $10 \mathrm{~s}$ and store at $-20^{\circ} \mathrm{C}\left(-80^{\circ} \mathrm{C}\right.$ for long-term storage).
3. Prepare the primer mix (10x) according to a worksheet (Table 2). Add appropriate volumes of primer stock solutions and sterile molecular grade water into a microcentrifuge tube. Mix all reagents well by vortexing for $10 \mathrm{~s}$.

\begin{tabular}{|c|c|c|c|}
\hline Component & Stock conc. $(\boldsymbol{\mu M})$ & Primer mix conc. $(\boldsymbol{\mu M})$ & Volume $(\boldsymbol{\mu L})$ \\
\hline Sal4-F3 primer & 100 & 1 & 10 \\
\hline Sal4-B3 primer & 100 & 1 & 10 \\
\hline Sal4-FIP primer & 100 & 18 & 180 \\
\hline
\end{tabular}




\begin{tabular}{|c|c|c|c|}
\hline Sal4-BIP primer & 100 & 18 & 180 \\
\hline Sal4-LF primer & 100 & 10 & 100 \\
\hline Sal4-LB primer & 100 & N/A & 420 \\
\hline Molecular grade water & N/A & N/A & 1000 \\
\hline Total & N/A & 100 \\
\hline
\end{tabular}

Table 2: Worksheet for preparing the LAMP primer mix (10x). The primers are listed in Table 1.

4. Aliquot the $10 \mathrm{x}$ primer mix to $500 \mu \mathrm{L}$ per microcentrifuge for preparing the LAMP master mix and adding DNA tube and store at $-20^{\circ} \mathrm{C}$. templates. Figure 1 is a LAMP diagram.

\section{Assembly of a LAMP reaction}

NOTE: To prevent cross-contamination, it is highly recommended to physically separate the areas used

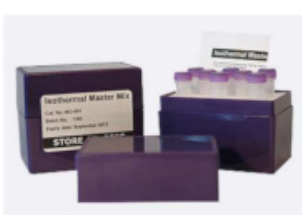

Prepare master mix

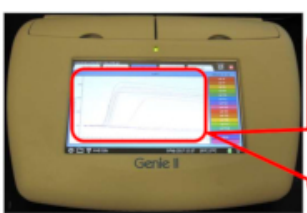

Run LAMP

(amplification graph generated in real-time)

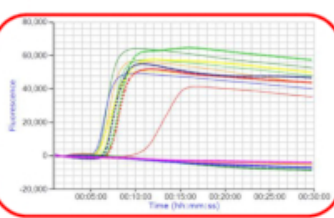

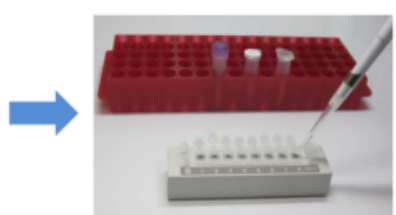

Distribute to wells

*DNA templates can be prepared from enrichment broths of animal food samples for screening purpose or cultures of presumptive Salmonella isolates for confirmation purpose.

Figure 1: A schematic diagram of the Salmonella LAMP workflow. Please click here to view a larger version of this figure.

1. Preparation and run setup

1. Clean bench with isopropanol and a DNA- and DNase-degrading solution (Table of Materials). 
Clean pipettes and tube strip holders (Table of Materials) with the DNA- and DNase-degrading solution.

2. Thaw the isothermal master mix, primer mix (10x), molecular grade water, positive control DNA, and DNA templates at room temperature.

3. Turn on the LAMP instrument (Table of Materials) and tap the opening screen to access the home screen. Follow these steps to create a run.

NOTE: One model of the LAMP instrument has 2 blocks ( $A$ and $B$ ) with 8 samples in each block and another model has a single block that accommodates 8 samples (Table of Materials).

1. Tap LAMP+Anneal and select Edit to enter sample information.

NOTE: The default LAMP run profile consists of amplification at $65{ }^{\circ} \mathrm{C}$ for $30 \mathrm{~min}$ and an anneal phase from $98{ }^{\circ} \mathrm{C}$ to $80^{\circ} \mathrm{C}$ with $0.05^{\circ} \mathrm{C}$ decrement per sec.

2. Tap each sample row to activate the cursor and enter relevant sample information, using the $\mathbf{A B}$ block icon to switch between the two LAMP instrument blocks.

3. Tap the Check icon when all sample information has been entered.

NOTE: Optionally, the run setup (termed "Profile," which contains sample information along with the default LAMP run profile) may be saved for later use. Tap the Save icon and give the profile a unique name. When testing this same set of samples next time, a new run can be initiated using the saved profile. Tap the Folder icon at the bottom left of the home screen and select Profile to load saved profiles.

2. LAMP reaction assembly

NOTE: When using both LAMP instrument blocks (A and $B$, a total of 16 samples), prepare the LAMP master mix for 18 samples. If using only one LAMP instrument block (8 samples total), prepare the LAMP master mix for 10 samples. For other sample numbers, adjust the volume accordingly to accommodate pipetting loss. Always include a positive control and an NTC in every LAMP run. Duplicate testing of each sample in independent LAMP runs is recommended.

1. Prepare the LAMP master mix according to a worksheet (Table 3). Add appropriate volumes of the isothermal master mix, primer mix, and molecular grade water into a microcentrifuge tube and vortex gently for $3 \mathrm{~s}$. Centrifuge briefly.

2. Place the tube strip in the strip holder and distribute $23 \mu \mathrm{L}$ of the LAMP master mix to each well.

3. Vortex all DNA templates and centrifuge briefly. Add $2 \mu \mathrm{L}$ of DNA template to the appropriate well and cap tightly.

4. Remove the tube strip from the holder and flick wrist to ensure all reagents have pooled at the bottom of the tube.

5. Load the tube strip into the LAMP instrument block(s), ensuring caps are secure before closing the lid.

\begin{tabular}{|c|c|c|c|c|c|}
\hline Component & Working conc. & $\begin{array}{c}\text { Final reaction } \\
\text { conc. }\end{array}$ & $\begin{array}{c}\text { Volume per } \\
\text { sample }(\mu \mathrm{L})\end{array}$ & $\begin{array}{c}\text { Volume for } 18 \\
\text { samples }(\mu \mathrm{L})\end{array}$ & $\begin{array}{c}\text { Volume for } 10 \\
\text { samples }(\mu \mathrm{L})\end{array}$ \\
\hline
\end{tabular}




\begin{tabular}{|c|c|c|c|c|c|}
\hline $\begin{array}{c}\text { ISO-001 isothermal } \\
\text { master mix }\end{array}$ & $1.67 x$ & $1 \mathrm{x}$ & 15 & 270 \\
\hline Primer mix & $10 \mathrm{x}$ & $1 \mathrm{x}$ & 2.5 & 45 & 25 \\
\hline $\begin{array}{c}\text { Molecular } \\
\text { grade water }\end{array}$ & N/A & N/A & 5.5 & 99 & 55 \\
\hline Master mix subtotal & N/A & N/A & 23 & 414 & 230 \\
\hline DNA template & N/A & N/A & 2 & N/A & N/A \\
\hline
\end{tabular}

Table 3: Worksheet for preparing the LAMP reaction mix. The primer mix (10x) is prepared according to Table 2 using stock solutions of primers listed in Table 1.

\section{LAMP Run}

NOTE: During a LAMP run, fluorescence readings are acquired using the FAM channel. The time-to-peak values ( $\left.T_{\max } ; \min \right)$ are determined automatically by the instrument for the time point when fluorescence ratio reaches the maximum value of the amplification rate curve. The $T_{m}\left({ }^{\circ} \mathrm{C}\right)$ is the melting/annealing temperature of the final amplified product.

1. Click on the Run icon at the upper right of the screen and select the block(s) containing tube strip(s) to start the LAMP run.

2. Optionally, while the reaction is in progress, tap the Temperature, Amplification, and Anneal tabs to see dynamic changes of various parameters during the LAMP run.

3. Once the run is complete, tap the Amplification and Anneal tabs to see complete amplification and anneal curves and tap the Results tab to view the results.
4. Optionally, for record keeping, record the run number located at the top left of the screen, using the format of "instrument serial number_run number," e.g., "GEN2-2209_0030."

\section{Interpretation of LAMP Results}

NOTE: LAMP results can be viewed on the LAMP instrument panel directly and/or using a LAMP software (Table of Materials).

1. To interpret LAMP results on the instrument panel, follow these steps.

1. Tap the Folder icon at the bottom left of the home screen and select Log to navigate to the file location to load the LAMP run of interest.

NOTE: The LAMP runs are organized by date, starting with year.

2. Observe the five tabs associated with each run: Profile, Temperature, Amplification, Anneal, and Results. 
NOTE: The Profile and Temperature tabs show programmed and actual temperatures, respectively, in the sample wells as the LAMP reaction proceeds. The Amplification and Anneal tabs show fluorescence readings and changes in fluorescence during the amplification and anneal phases, respectively. The Results tab shows a tabular view of the LAMP results.

3. Tap the Results tab to observe LAMP results for each well.

NOTE: There are three columns (Well, Amplification, and Anneal). The "Amplification" column shows the time-to-peak values ( $T_{\max }$; min:sec) for each sample ("Well") and the "Anneal" column shows the melting/annealing temperatures $\left(T_{m} ;{ }^{\circ} \mathrm{C}\right)$ for any amplified product in that well.

4. Interpret the LAMP results and report final LAMP results as follows.

1. Examine the control wells first. The NTC well should have blank $T_{\max }$ while $T_{m}$ can be either blank (both LAMP instrument models) or $<83$ ${ }^{\circ} \mathrm{C}$ (only for the LAMP instrument model with two blocks). The positive control well should have $T_{\text {max }}$ between 5 and 10 min and $T_{m}$ around 90 ${ }^{\circ} \mathrm{C}$.

2. Examine the sample wells. All samples with the correct $T_{m}$ (approximately $90{ }^{\circ} \mathrm{C}$ ) and $T_{\max }$ (between 5-30 $\mathrm{min}$ ) are considered positive for Salmonella.

3. Report final LAMP results based on results from duplicate runs. If the duplicate runs have consistent results, final LAMP results can be reported. If duplicate runs are inconsistent, repeat both runs independently. If results are still inconsistent, the sample should be considered presumptive positive for Salmonella and will need to go through culture confirmation.

2. To interpret LAMP results using the software, follow these steps.

1. Click on the Computer icon on the left panel and navigate to the file location to load the LAMP run of interest.

NOTE: The computer with the software installed does not need to be connected to the LAMP instrument to analyze LAMP results, i.e., remote access is available. The LAMP runs are organized by date.

2. Observe the seven tabs associated with each run: Profile, Temperature, Amplification, Amplification Rate, Anneal, Anneal Derivative, and Result.

NOTE: Similar to the instrument panel view, the Profile and Temperature tabs show programmed and actual temperatures, respectively, in the sample wells as the LAMP reaction proceeds. The Amplification/Amplification Rate and Anneal/Anneal Derivative tabs show fluorescence readings or changes in fluorescence during the amplification and anneal phases, respectively. The Results tab shows a tabular view of the LAMP results which differ slightly from the instrument panel view.

3. Tap the Amplification Rate tab to view a graphic display of the fluorescence ratios by time. Click on the Setting icon at the top right of the screen and 
adjust the "Peak Detection Threshold Ratio" from 0.020 to 0.010 .

NOTE: The adjustment is necessary to ensure that all valid peaks are identified, and the results obtained using the software match with those displayed on the instrument panel.

4. Tap the Result tab to observe LAMP results for each well.

NOTE: There are four columns (Graph Name, Well Number, Well Name, and Peak Value). The top portion of the "Peak Value" column shows "Amp Time" ( $\left.T_{\max } ; \min : \mathrm{sec}\right)$ for each sample ("Well Name") while the bottom portion shows "Anneal Derivative" $\left(T_{m} ;{ }^{\circ} \mathrm{C}\right)$ for any amplified product in that well.

5. Interpret the LAMP results and report final LAMP results following similar steps as when using the instrument panel with one exception that the NTC well and other negative samples should have blank $T_{m}$ as the LAMP software settings eliminate those $T_{m}<83{ }^{\circ} \mathrm{C}$ results. Similarly, all samples with the correct $T_{m}$ (approximately $90{ }^{\circ} \mathrm{C}$ ) and $T_{\max }$ (between 5-30 $\mathrm{min}$ ) are considered positive for Salmonella.

\section{Representative Results}

Figure 2 and Figure 3 show representative LAMP graphs/ tables displayed on both platforms. In this LAMP run, samples S1 to S6 are 10-fold serial dilutions of S. enterica serovar Infantis ATCC 51741 ranging from $1.1 \times 10^{6} \mathrm{CFU}$ to 11 CFU per reaction. Positive control is $S$. enterica serovar Typhimurium ATCC 19585 (LT2) at $1.7 \times 10^{4}$ CFU per reaction and NTC is molecular grade water.

As shown in Figure 2E and Figure 3G, both NTC and PC wells are valid controls. The NTC well has blank $T_{\max }$ while $T_{m}$ is $<83^{\circ} \mathrm{C}$ on the LAMP instrument panel and blank in the LAMP software, suggesting a negative result. The PC well has $T_{\max }$ of $7 \min 45 \mathrm{sec}$ and $T_{m}$ of $\sim 90{ }^{\circ} \mathrm{C}$ on both platforms, suggesting a positive result. Samples S1 to S6 have $T_{\max }$ between $6 \mathrm{~min} 30 \mathrm{sec}$ and $12 \mathrm{~min} 15 \mathrm{sec}$, all being Salmonella-positive.

Following duplicate runs of the same set of samples, the final LAMP results are reported for these samples. This representative LAMP run shows that LAMP successfully detects Salmonella with a wide range of concentrations in the samples. 


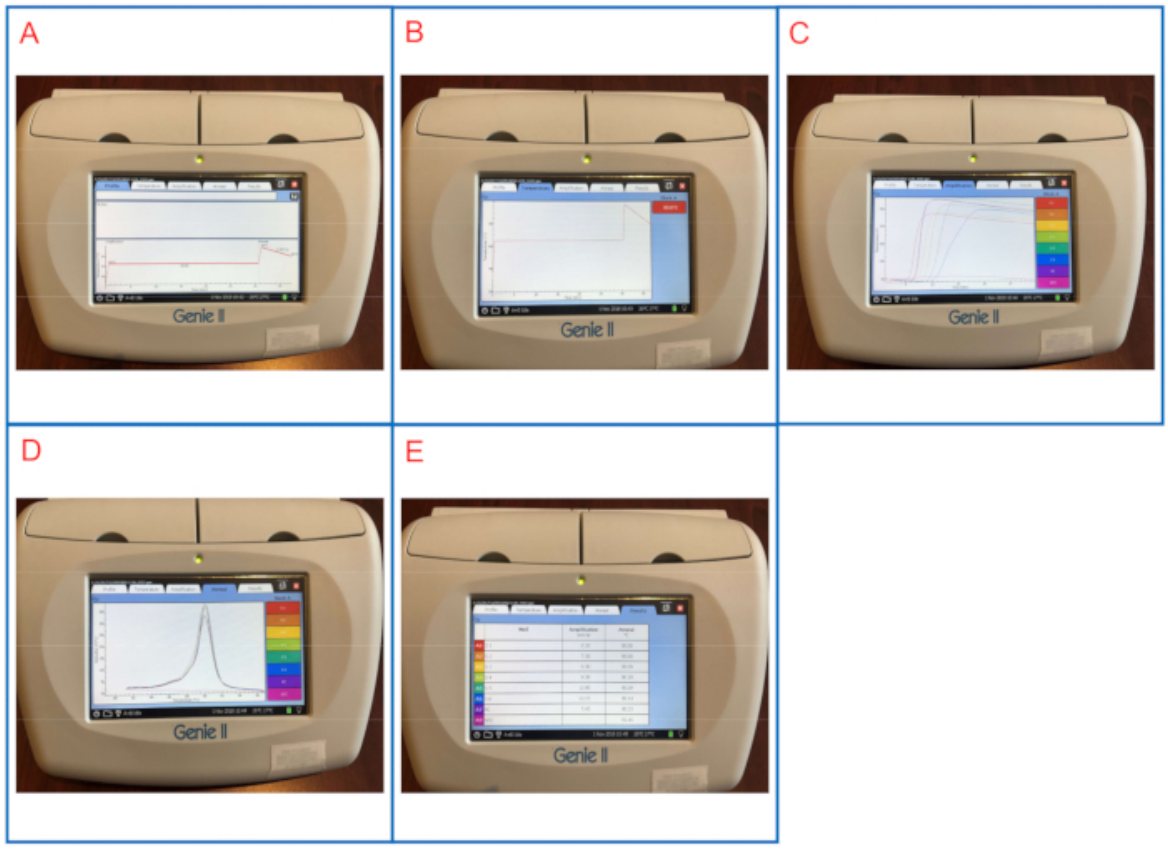

Figure 2: Representative LAMP results displayed on the LAMP instrument panel. (A) The Profile tab shows the programmed temperature profile. (B) The Temperature tab shows actual temperatures in the sample wells as LAMP reaction proceeds. (C) The Amplification tab shows fluorescence readings during LAMP amplification. (D) The Anneal tab shows changes in fluorescence (derivative) during the anneal phase. (E) The Results tab shows a tabular view of the LAMP results. Please click here to view a larger version of this figure. 


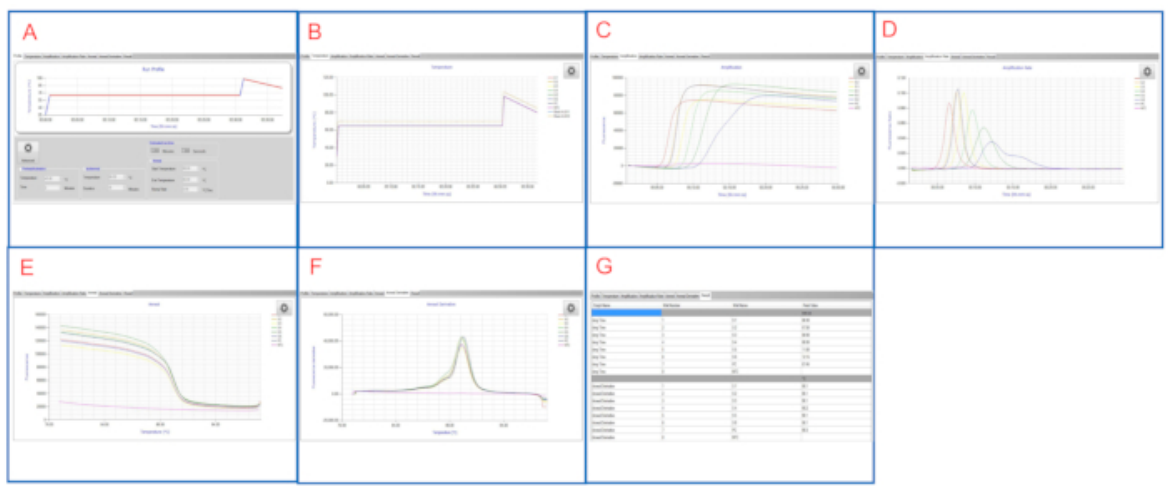

Figure 3: Representative LAMP results viewed in the LAMP software. (A) The Profile tab shows the programmed temperature profile. (B) The Temperature tab shows actual temperatures in the sample wells as LAMP reaction proceeds. (C) The Amplification tab shows fluorescence readings during LAMP amplification. (D) The Amplification Rate tab shows changes in fluorescence (fluorescence ratio) during LAMP amplification. (E) The Anneal tab shows fluorescence readings during the anneal phase. (F) The Anneal Derivative tab shows changes in fluorescence (derivative) during the anneal phase. (G) The Result tab shows a tabular view of the LAMP results. Please click here to view a larger version of this figure.

\section{Discussion}

We have presented here a simple, rapid, specific, and sensitive LAMP method for screening and confirming Salmonella in animal food and pure culture, respectively. With the convenience of an isothermal master mix that contains four key reagents, and a ready-to-use, in-house prepared primer mix, assembling a LAMP reaction requires only a few pipetting steps (Figure 1). The total run time including amplification and anneal phases is less than 38 min (Figure 2A,B and Figure 3A,B). Positive results are monitored via real-time fluorescence (Figure 2C and Figure 3C,D) and can be detected as early as $5 \mathrm{~min}^{26}$. The anneal phase serves as an extra confirmation of LAMP specificity since only samples with correct $T_{m}$ (around $90^{\circ} \mathrm{C}$ ) are reported as positive (Figure 2D,E and Figure 3E-G). Sensitivities of 1 Salmonella cell in pure culture and $<1 \mathrm{CFU} / 25 \mathrm{~g}$ in animal food have been reported previously 26 .
As LAMP is quite effective and generates a large quantity of $D N A^{1}$, it is critical that best laboratory practices are used to prevent cross-contamination, which may include physically separating the areas for preparing the LAMP master mix and adding DNA templates, avoiding generating aerosols, using filter pipette tips, changing gloves often, and refraining from opening LAMP reaction tubes post-amplification.

The specificity of this Salmonella LAMP method was previously tested using 300 bacterial strains (247 Salmonella of 185 serovars and 53 non-Salmonella) and demonstrated to be $100 \%$ specific $^{26}$. Notably, significant differences in $T_{\max }$ were observed between the two Salmonella species, S. enterica and Salmonella bongori, and among S. enterica subspecies, especially subsp. arizonae (IIla $)^{26}$. Nonetheless, these were still valid positive results per the rules for interpreting LAMP results. In our multi-laboratory collaborative study in dry dog food which involved 14 
analysts ${ }^{19}$, samples having inconsistent results in duplicate LAMP runs were occasionally observed. These usually involved samples with delayed positive results $\left(T_{\max }>15\right.$ min). Repeating both runs independently usually resolved the issue. More rarely, we observed samples with correct $T_{m}$ but no or irregular $T_{\max }$ values ( $\left.<5 \mathrm{~min}\right)$. This was usually caused by air bubbles in the reaction tube.

Throughout the lifecycle of LAMP method development, evaluation, precollaborative study, and multi-laboratory validation, we have observed high tolerance of LAMP to inhibitors in various animal food or food matrices and culture media $4,19,22,23,24$, highlighting the robustness of the method and collaborating numerous other studies on a global scale ${ }^{8}$. This is superior compared to PCR or real-time PCR, which usually requires an internal amplification control to ensure that negative results are not due to matrix inhibition ${ }^{28}$. Further, LAMP demonstrated similar (or superior) specificity and sensitivity compared to PCR or real-time PCR in the vast majority of studies ${ }^{8}$. The cost of LAMP reagents is at about $\$ 1$ per reaction. The LAMP instruments used in this protocol are small, low-maintenance, and portable. They can handle any isothermal amplification method that employs target detection by fluorescence measurement, LAMP included. Using the LAMP software, comprehensive reports can be generated in multiple format (pdf, text, and image).

Method validation is a critical step before a new method can be adopted for routine use. It is noteworthy that the LAMP protocol reported here has successfully completed multi-laboratory validation ${ }^{19}$. With the recent incorporation of this LAMP protocol into the U.S. FDA's BAM Chapter 5 Salmonella ${ }^{27}$, it is expected that the method will gain much wider use, both as a rapid screening method in animal food and as a reliable confirmation method for presumptive Salmonella isolates from all food categories.

\section{Disclosures}

The authors declare that they have no competing financial interests. The views expressed in this manuscript are those of the authors and do not necessarily reflect the official policy of the Department of Health and Human Services, the U.S. Food and Drug Administration, or the U.S. Government. Reference to any commercial materials, equipment, or process does not in any way constitute approval, endorsement, or recommendation by the Food and Drug Administration.

\section{Acknowledgments}

The authors thank members of the FDA's Microbiology Methods Validation Subcommittee (MMVS) and Bacteriological Analytical Manual (BAM) Council for critically reviewing Salmonella LAMP method validation studies.

\section{References}

1. Notomi, T. et al. Loop-mediated isothermal amplification of DNA. Nucleic Acids Research. 28 (12), E63 (2000).

2. Kaneko, H., Kawana, T., Fukushima, E., Suzutani, T. Tolerance of loop-mediated isothermal amplification to a culture medium and biological substances. Journal of Biochemical and Biophysical Methods. 70 (3), 499-501 (2007).

3. Francois, P. et al. Robustness of a loopmediated isothermal amplification reaction for diagnostic applications. FEMS Immunology and Medical Microbiology. 62 (1), 41-48 (2011). 
4. Yang, Q., Wang, F., Prinyawiwatkul, W., Ge, B. Robustness of Salmonella loop-mediated isothermal amplification assays for food applications. Journal of Applied Microbiology. 116 (1), 81-88 (2014).

5. Nagamine, K., Watanabe, K., Ohtsuka, K., Hase, T., Notomi, T. Loop-mediated isothermal amplification reaction using a nondenatured template. Clinical Chemistry. 47 (9), 1742-1743 (2001).

6. Zhang, X., Lowe, S. B., Gooding, J. J. Brief review of monitoring methods for loop-mediated isothermal amplification (LAMP). Biosensors \& Bioelectronics. 61 491-499 (2014).

7. Nagamine, K., Hase, T., Notomi, T. Accelerated reaction by loop-mediated isothermal amplification using loop primers. Molecular and Cellular Probes. 16 (3), 223-229 (2002).

8. Yang, Q., Domesle, K. J., Ge, B. Loop-mediated isothermal amplification for Salmonella detection in food and feed: Current applications and future directions. Foodborne Pathogens and Disease. 15 (6), 309-331 (2018).

9. Mori, Y., Notomi, T. Loop-mediated isothermal amplification (LAMP): Expansion of its practical application as a tool to achieve universal health coverage. Journal of Infection and Chemotherapy. 26 (1), 13-17 (2020).

10. Mansour, S. M., Ali, H., Chase, C. C., Cepica, A. Loopmediated isothermal amplification for diagnosis of 18 World Organization for Animal Health (OIE) notifiable viral diseases of ruminants, swine and poultry. Animal Health Research Reviews. 16 (2), 89-106 (2015).
11. Kumar, Y., Bansal, S., Jaiswal, P. Loop-mediated isothermal amplification (LAMP): $A$ rapid and sensitive tool for quality assessment of meat products. Comprehensive Reviews in Food Science and Food Safety. 16 (6), 1359-1378 (2017).

12. WHO. The use of loop-mediated isothermal amplification (TB-LAMP) for the diagnosis of pulmonary tuberculosis: policy guidance. http://www.who.int/tb/ publications/lamp-diagnosis-molecular/en/ (2016).

13. Kundapur, R. R., Nema, V. Loop-mediated isothermal amplification: Beyond microbial identification. Cogent Biology. 2, 1137110 (2016).

14. WHO. Salmonella (non-typhoidal) fact sheet. http:// www.who.int/mediacentre/factsheets/fs139/en/ (2018).

15. FAO/WHO. Executive summary report of the joint FAO/ WHO expert meeting on hazards associated with animal feed. http://www.fao.org/3/a-az851e.pdf (2015).

16. FDA. Compliance Policy Guide Sec. 690.800 Salmonella in Food for Animals. https://www.fda.gov/media/86240/ download (2013).

17. Bird, $\mathrm{P}$. et al. Evaluation of the $3 \mathrm{M}$ molecular detection assay (MDA) 2 - Salmonella for the detection of Salmonella spp. in select foods and environmental surfaces: collaborative study, first action 2016.01. Journal of AOAC International. 99 (4), 980-997 (2016).

18. D'Agostino, M. et al. Validation of a loop-mediated amplification/ISO 6579-based method for analysing soya meal for the presence of Salmonella enterica. Food Analytical Methods. 9 (11), 2979-2985 (2016).

19. Ge, B. et al. Multi-laboratory validation of a loopmediated isothermal amplification method for screening 
Salmonella in animal food. Frontiers in Microbiology. 10, 562 (2019).

20. D'Agostino, M., Diez-Valcarce, M., Robles, S., LosillaGarcia, B., Cook, N. A loop-mediated isothermal amplification-based method for analysing animal feed for the presence of Salmonella. Food Analytical Methods. 8 (10), 2409-2416 (2015).

21. Galan, J. E., Ginocchio, C., Costeas, P. Molecular and functional characterization of the Salmonella invasion gene invA: homology of InvA to members of a new protein family. Journal of Bacteriology. 174 (13), 4338-4349 (1992).

22. Chen, S., Wang, F., Beaulieu, J. C., Stein, R. E., Ge, B. Rapid detection of viable salmonellae in produce by coupling propidium monoazide with loop-mediated isothermal amplification. Applied and Environmental Microbiology. 77 (12), 4008-4016 (2011).

23. Yang, Q., Chen, S., Ge, B. Detecting Salmonella serovars in shell eggs by loop-mediated isothermal amplification. Journal of Food Protection. 76 (10), 1790-1796 (2013).

24. Yang, Q. et al. Evaluation of loop-mediated isothermal amplification for the rapid, reliable, and robust detection of Salmonella in produce. Food Microbiology. 46, 485-493 (2015).

25. Yang, Q., Domesle, K. J., Wang, F., Ge, B. Rapid detection of Salmonella in food and feed by coupling loop-mediated isothermal amplification with bioluminescent assay in real-time. BMC Microbiology. 16 (1), 112 (2016).

26. Domesle, K. J., Yang, Q., Hammack, T. S., Ge, B. Validation of a Salmonella loop-mediated isothermal amplification assay in animal food. International Journal of Food Microbiology. 264 63-76, (2018).

27. Andrews, W. H., Jacobson, A., Hammack, T. S. Bacteriological Analytical Manual. Chapter 5: Salmonella. http://www.fda.gov/Food/ FoodScienceResearch/LaboratoryMethods/ ucm070149.htm (2020).

28. Bustin, S. A. et al. The MIQE guidelines: minimum information for publication of quantitative real-time PCR experiments. Clinical Chemistry. 55 (4), 611-622 (2009). 